\title{
Absolute leukocytes count and NLR as a diagnostic and prognostic biomarkers for severity of COVID-19 infection
}

\author{
Khalid Abdelsamea Mohamedahmed $\mathbb{C}^{1,2^{*}}$, Adam Dawoud Abakar ${ }^{3}$ \\ 1 Department of Hematology, Faculty of Medical Laboratory Science, \\ University of Gezira, Wad Medani, Sudan \\ 2 Department of Immunology, Faculty of Medical Laboratory Science, \\ University of Gezira, Wad Medani, Sudan \\ 3 Department of Medical Parasitology, Faculty of Medical Laboratory \\ Science, University of Gezira, Wad Medani, Sudan
}

The current COVID-19 pandemic challenges not only the lack of awareness of the disease, but also the rapid diagnosis, the prediction of severe early disease, the clinical characterization of mortality and severity of patients, and the effective management that affects the global scale, resulting in low capacities of healthcare systems, the resilience of systems and global economics. Therefore, early differential diagnosis and prediction of SARS-COV-2 infection severity are needed. CRP and NLR are diagnostic marker commonly used, most available, effective, and economically used primarily to evaluate the ongoing systemic inflammatory response. By developing the rapid diagnosis of SARS-CoV-2 using RT-PCR, the diagnosis of SARS-CoV-2 was facilitated by WHO network laboratory protocols. ${ }^{1}$ RT-PCR results can give false negatives depending on the test, sample, and period of the disease. $\stackrel{?}{2}$

Elevated CRP values have been previously reported in patients with COVID-19 during hospitalization with higher ICU patient levels. ${ }^{3}$ Recent study concluded that CRP was a predictive and prognostic marker for early severe COVID-19. ${ }^{4}$ An innate immune response is the first-line for clearance of viral infections, CRP might be linked to the overproduction of inflammatory cytokines in severe patients and may lead to dysfunction of various organ systems in COVID-19-infected patients. ${ }^{5}$

Most patients with extremely extreme COVID-19 types (ICU) show that deregulation of the immune system results in a rapid decrease in the number of lymphocytes (lymphopenia) and a rise in the number of neutrophils (neutrophilia). ${ }^{6}$ Although lymphopenia is an early marker, neutrophilia is a COVID-19 late-onset maker. ${ }^{-}$The proposed lymphopenia mechanisms are either chemokine-mediated redistribution and sequestration of lymphocytes in the lungs or suppression of the bone marrow via CD13 or CD66; the most powerful suggested mechanism is the condition of 'hypercytokinemia' or cytokine storm, demonstrated in COVID-19 patien. ${ }^{7}$ Neutrophil Lymphocyte Ratio (NLR) is a commonly used marker for evaluating the prognosis of cancer, inflammatory and infectious diseases in patients and more accurately reflects the balanced relationship between the severity of inflammatory reactions and the immune

\footnotetext{
Corresponding author

E-mail address: khalid.abdelsamea@hotmail.com (Khalid AM)
} 
state. ${ }^{8}$ Despite a significantly higher NLR value in severe patients on admission, non-ICU patients remain borderline NLR value during hospitalization. $, \underline{6}, \underline{10}$

Recent studies found that during the entire hospital stay, NLR correlates well with severity (significantly increased at week 2 (threshold) and significantly decreases down by week 3$). \stackrel{10}{-}$ Several studies have shown that the level of CRP and the value of NLR are independent risk factors for severe COVID-19 and have concluded that high NLR and CRP can serve as an early marker of severe COVID-19. $\underline{6} . \underline{9}$

This observation illustrates the fact that it can be useful to combine the NLR value with the level of CRP as well as lymphopenia and neutrophilia to predict terrible outcomes at admission. In addition to rapid intervention, we concluded early detection and risk stratification of COVID-19 by warning signs within clear and convenient predictive measures (high values of CRP, NLR, as well as lymphopenia and neutrophilia) to guide clinical practice will greatly reduce mortality, improve the rate of cure and shorten the period of hospital stay.

\section{AUTHORS' CONTRIBUTIONS}

All authors contributed equally to this work.

\section{FOUNDING INFORMATION}

This research received no specific grant from any funding agency in the public, commercial or not-for-profit sectors.

\section{DATA AVAILABILITY STATEMENT}

Data sharing is not applicable to this article as no new data were created or analyzed in this study.

\section{DISCLOSURE STATEMENT}

The views and opinions expressed in this article are those of the authors and do not necessarily reflect the official policy or position of any affiliated agency of the authors.

\section{REFERENCES}

1. Organization WH. Laboratory Testing for Coronavirus Disease 2019 (COVID-19) in Suspected Human Cases: Interim Guidance, 2 March 2020. World Health Organization https://apps.who.int/iris/handle/10665/331329

2. Wang $\mathrm{P}$, Anderson N, Pan Y, et al. The SARS-CoV-2 Outbreak: Diagnosis, Infection Prevention, and Public Perception. Clin Chem. 2020;66(5):644-651. doi:10.1093/clinchem/hvaa080

3. Chen W, Zheng KI, Liu S, Yan Z, Xu C, Qiao Z. Plasma CRP level is positively associated with the severity of COVID-19. Ann Clin Microbiol Antimicrob. 2020;19(1):18. doi:10.1186/s12941-020-00362-2

4. Tan C, Huang Y, Shi F, et al. C-reactive protein correlates with computed tomographic findings and predicts severe COVID-19 early. J Med Virol. 2020;92(7):856-862. doi:10.1002/jmv.25871

5. Feng Z, Yu Q, Yao S, et al. Early Prediction of Disease Progression in 2019 Novel Coronavirus Pneumonia Patients Outside Wuhan with CT and Clinical Characteristics. medRxiv. Published online January 1, 2020:2020.02.19.20025296. doi:10.1101/2020.02.19.20025296

6. Yufei Y, Mingli L, Xuejiao L, et al. Utility of the neutrophil-to-lymphocyte ratio and C-reactive protein level for coronavirus disease 2019 (COVID19). Scand J Clin Lab Invest. 2020;80(7):536-540. doi: $10.1080 / 00365513.2020 .1803587$

7. He Z, Zhao C, Dong Q, et al. Effects of severe acute respiratory syndrome (SARS) coronavirus infection on peripheral blood lymphocytes and their 
subsets. Int J Infect Dis. 2005;9(6):323-330. doi:10.1016/i.ijid.2004.07.014

8. Forget $\mathrm{P}$, Khalifa C, Defour J-P, Latinne D, Van Pel M-C, De Kock M. What is the normal value of the neutrophil-to-lymphocyte ratio? BMC Res Notes. 2017;10(1):12. doi:10.1186/s13104-016-2335-5

9. Khourssaji M, Chapelle V, Evenepoel A, et al. A biological profile for diagnosis and outcome of COVID-19 patients. Clin Chem Lab Med. 2020;58(12):2141-2150. doi:10.1515/cclm-2020-0626

10. Ponti G, Maccaferri M, Ruini C, Tomasi A, Ozben T. Biomarkers associated with COVID-19 disease progression. Crit Rev Clin Lab Sci. 2020;57(6):389-399. doi:10.1080/10408363.2020.1770685 